Calculation of rovibrational spectra of water by means of particles-on-concentricspheres models. II. Excited states of stretching vibrations

Grigory A. Natanson, Gregory S. Ezra, Gerardo Delgado-Barrio, and R. Stephen Berry

Citation: The Journal of Chemical Physics 84, 2035 (1986); doi: 10.1063/1.450411

View online: https://doi.org/10.1063/1.450411

View Table of Contents: http://aip.scitation.org/toc/jcp/84/4

Published by the American Institute of Physics

Articles you may be interested in

Calculation of rovibrational spectra of water by means of particles-on-concentric-spheres models. I.

Ground stretching vibrational state

The Journal of Chemical Physics 81, 3400 (1984); 10.1063/1.448091

PHYSICS TODAY WHITEPAPERS
ADVANCED LIGHT CURE ADHESIVES

READ NOW

Take a closer look at what these environmentally friendly adhesive systems can do
PRESENTED BY

Q MASTERBOND。 


\title{
Calculation of rovibrational spectra of water by means of particles-on- concentric-spheres models. II. Excited states of stretching vibrations
}

\author{
Grigory A. Natanson, ${ }^{\text {a) }}$ Gregory S. Ezra, ${ }^{\text {b) }}$ Gerardo Delgado-Barrio, ${ }^{\text {c) }}$ \\ and R. Stephen Berry \\ Department of Chemistry, The University of Chicago, Chicago, Illinois 60637
}

(Received 15 August 1985; accepted 7 November 1985)

\begin{abstract}
It is shown that adiabatic separation of high-frequency stretching modes from bending and overall rotational motions in triatomic molecules $\mathrm{XY}_{2}$ leads naturally to the particles-on-a-sphere (POS) model treated previously [J. Chem. Phys. 81, 3400 (1984)]. Solution of the rovibrational problem using a further approximation in which stretching motions are treated as uncoupled modes is then investigated in detail. It is shown that, for states with a significantly larger number of quanta in one bond than the other, the POS model in this approximation yields energy levels that are essentially identical with those for the particles-on-concentric-spheres (POCS) model, where the latter is obtained using a different decoupling of the basic set of differential equations.
\end{abstract}

\section{INTRODUCTION}

As shown in our previous paper (part I, Ref. 1), an adiabatic separation of fast unexcited stretching modes from bending and overall rotational motions in the water molecule leads naturally to an approach in which the two hydrogen atoms are treated as particles moving on a sphere. This model was used to calculate bending frequencies and rotational structure in the ground and excited bending states. Depending on the potential energy surface used, the accuracy of the resulting energy levels is comparable with or in some cases even better than that obtained with more conventional models of the water molecule, such as the rigid bend$\mathrm{er}^{2,3}$ or the semirigid bender ${ }^{4}$ modification suggested by Bunker and Landsberg.

The aim of the present work is to provide a more rigorous justification for the model used previously, and thereby to extend application of the method to states involving excitation of stretching modes as well as bending modes and rotations.

To the best of our knowledge, the only variational calculations to date of rotational spectra for the water molecule in local-mode stretching states are those recently reported by Reimers and Watts. ${ }^{5}$ For their calculations, these authors used a new surface consisting of three Morse-type potentials. They were able to fit their calculated energy levels to experimental vibrational frequencies with impressive accuracy using only nine parameters. However, we shall show below that this surface does not provide an accurate description of the effect of vibrational excitation on rotational structure.

We have now experienced comparable difficulties in describing the effects of vibration upon rotational structure in our own calculations, but in our case the main source of error is not the potential surface used ${ }^{6}$ but the dynamical approxi-

\footnotetext{
'Present address: Department of Chemistry, Northwestern University, Evanston, Illinois 60201.

b) Present address: Department of Chemistry, Baker Laboratory, Cornell University, Ithaca, New York 14853.

c) Present address: Institute de Estructura de la Materia, C.S.I.C., Serrano, 119, Madrid-6, Spain.
}

mations made in the Hamiltonian. In spite of these approximations we are nevertheless able to obtain qualitative regularities and trends in rotational bands somewhat better than do Reimers and Watts, ${ }^{5}$ who performed more accurate calculations with a more approximate potential surface.

\section{BASIC EQUATIONS}

We start with the laboratory-frame Hamiltonian used in part I:

$$
\widehat{H}=-\frac{1}{2 \mu}\left(\nabla_{1}^{2}+\nabla_{2}^{2}\right)-\frac{1}{M} \nabla_{1} \cdot \nabla_{2}+V\left(R_{1}, R_{2}, \gamma\right),
$$

where $R_{1}$ and $R_{2}$ are bond lengths, $\gamma$ is the included bending angle, $m$ and $M$ are masses of hydrogen and oxygen, respectively, $V\left(R_{1}, R_{2}, \gamma\right)$ is the Born-Oppenheimer potential, $\mu^{-1}=m^{-1}+M^{-1}$ and

$$
\nabla_{(i)}=\frac{\partial}{\partial \mathbf{R}_{i}},
$$

where the vector $\mathbf{R}_{i}$ is the position of hydrogen $i$ with respect to the oxygen atom.

\section{A. 2D stretch expansion}

We now treat the (fast) stretching modes by analogy with the treatment of electronic motion in the zeroth-order Born-Oppenheimer approximation. That is, we expand the full molecular rovibrational wave function as

$$
\Psi_{v, \tau}\left(\mathbf{R}_{1}, \mathbf{R}_{2}\right)=\frac{1}{\boldsymbol{R}_{1} \boldsymbol{R}_{2}} \sum_{\tilde{v}} \Phi_{\tilde{v}}\left(\boldsymbol{R}_{1}, \boldsymbol{R}_{2}\right) \chi_{v}^{\tilde{v}, \tau}\left(\hat{\mathbf{R}}_{1}, \hat{\mathbf{R}}_{2}\right),
$$

where $\hat{\mathbf{R}}_{i} \equiv \mathbf{R}_{i} / R_{i}$, and the function $\Phi_{v}$ is a solution of the two-dimensional problem ${ }^{6}$

$$
\begin{aligned}
& {\left[-\frac{1}{2 \mu}\left(\frac{\partial^{2}}{\partial R_{1}^{2}}+\frac{\partial^{2}}{\partial R_{2}^{2}}\right)-\frac{1}{M} \cos \gamma_{e} \frac{\partial^{2}}{\partial R_{1} \partial R_{2}}\right.} \\
& \left.\quad+V\left(R_{1}, R_{2}, \gamma_{e}\right)-E_{v}^{(2 D)}\right] \Phi_{v}\left(R_{1}, R_{2}\right)=0,
\end{aligned}
$$

which is normalized as follows:

$$
\int \Phi_{v}^{2} d R_{1} d R_{2}=1
$$


Note the presence of the equilibrium bending angle $\gamma_{e}$ in Eq. (4). Indices $v$ and $v, \tau$ denote complete sets of quantum numbers necessary to specify solutions of the two-dimensional stretching and full rotation-vibration problems, respectively. In particular,

$$
v=n, m, \kappa \text {, }
$$

where $n$ and $m(n<m)$ are the numbers of quanta of stretching excitation in each bond, and $\kappa$ is \pm 1 depending upon whether the stretching function $\Phi_{v}\left(R_{1} R_{2}\right)$ is symmetric $(+1)$ or antisymmetric $(-1)$ with respect to permutation of $R_{1}$ and $R_{2}$. Following Lawton and Child ${ }^{6}$ we shall also use the notation

$$
v=n m^{[\kappa]},
$$

where $[\kappa] \equiv \operatorname{sign}[\kappa]$. The set of quantum numbers $\tau$ includes $v_{2}$, the number of bending quanta, $J$, the total angular momentum, and $\left(K_{a}, K_{c}\right)$, rotational quantum numbers corresponding to prolate and oblate symmetric-top quantization.

Substituting the expansion (3) into the Schrödinger equation (1), multiplying the resultant expression by $R_{1} R_{2} \cdot \Phi_{v^{\prime}}\left(R_{1} R_{2}\right)$, and integrating over $R_{1}$ and $R_{2}$, taking into account the fact that the $\Phi_{v}\left(R_{1} R_{2}\right)$ form an orthonormal set, we obtain a set of coupled differential equations for the rotation-bending functions $\chi_{v, \tau}^{v^{\prime}}$ :

$$
\begin{aligned}
& {\left[\hat{t}\left(I_{\mu}^{\left(v^{\prime}\right)}, I_{\mathcal{M}^{\prime}}^{\left(v^{\prime}\right)}\right)+W_{v^{\prime}}(\gamma)-\delta_{v v^{\prime}} E_{v, \tau}\right] \chi_{v, \tau}^{v^{\prime}}} \\
& \quad=\sum_{v^{\prime} \neq v^{\prime}}\left[a_{v^{\prime} ; v^{\prime \prime}}\left(\Delta_{\theta_{1} \varphi_{1}}+\kappa^{\prime} \kappa^{\prime \prime} \Delta_{\theta_{2} \varphi_{2}}\right)+b_{v^{\prime} ; v^{\prime \prime}} \nabla_{\theta_{1} \varphi_{1}} \cdot \nabla_{\theta_{2} \varphi_{2}}\right. \\
& \left.\quad+c_{v^{\prime} v^{\prime \prime}}\left(\hat{\mathbf{R}}_{1} \cdot \nabla_{\theta_{2} \varphi_{2}}+\kappa^{\prime} \kappa^{\prime \prime} \hat{\mathbf{R}}_{2} \cdot \nabla_{\theta_{1} \varphi_{1}}\right)-w_{v^{\prime} ; v^{\prime \prime}}(\gamma)\right] \chi_{v, r}^{v^{*},},
\end{aligned}
$$

where

$$
\hat{t}\left(I_{\mu}, I_{M}\right) \equiv \hat{t}_{0}\left(I_{\mu}\right)+\hat{t}_{1}\left(I_{M}\right),
$$

the adiabatic contribution to all the angular parts of the kinetic energy;

$$
\hat{t}_{0}(I) \equiv-\frac{1}{2 I}\left(\Delta_{\theta_{1} \varphi_{1}}+\Delta_{\theta_{2} \varphi_{2}}\right),
$$

the (presumably large) uncoupled parts of the angular kinetic energy for individual hydrogens;

$$
\hat{t}_{1}(I) \equiv \Delta \hat{t}(I)+\Delta \hat{t}^{\prime}(I)
$$

all the adiabatic kinetic angular couplings;

$$
\hat{\Delta t}(I) \equiv-\frac{1}{I} \nabla_{\theta_{1} \varphi_{1}} \cdot \nabla_{\theta_{2} \varphi_{2}},
$$

the angular coupling through the center-of-mass momenta correction in the POCS model;

$$
\Delta t^{\prime}(I) \equiv \frac{1}{I}\left(\hat{R}_{1} \cdot \nabla_{\theta_{2} \varphi_{2}}+\hat{R}_{2} \cdot \nabla_{\theta_{1} \varphi_{1}}\right),
$$

the adiabatic correction to the POCS model;

$$
I_{\mu}^{(v)} \equiv\left[\frac{1}{\mu} \int R_{1}^{-2} \Phi_{v}^{2}\left(R_{1}, R_{2}\right) d R_{1} d R_{2}\right]^{-1} \equiv \mu\left[\bar{R}^{(v)}\right]^{2},
$$

the effective moment of inertia of the light particle;

$$
\begin{aligned}
I_{M}^{(v)} & \equiv\left[\frac{1}{M} \int R_{1}^{-1} R_{2}^{-1} \Phi_{v}^{2}\left(R_{1}, R_{2}\right) d R_{1} d R_{2}\right]^{-1} \\
& \equiv M^{(v)}\left[\bar{R}^{(v)}\right]^{2}
\end{aligned}
$$

the effective moment of inertia of angular couplings; with

$M^{(v)} \equiv M\left[\int R_{1}^{-1} R_{2}^{-1}\left(\bar{R}^{(v)}\right)^{2} \Phi_{v}^{2}\left(R_{1}, R_{2}\right) d R_{1} d R_{2}\right]^{-1}$,

the effective mass of the heavy particle;

$$
\begin{aligned}
& W_{v^{\prime}}(\gamma) \\
& \equiv E_{v^{\prime}}^{(2 \mathrm{D})}+\int\left[V\left(R_{1}, R_{2}, \gamma\right)-V\left(R_{1}, R_{2}, \gamma_{e}\right)\right] \Phi_{v^{\prime}}^{2} d R_{1} d R_{2} \\
& \quad-\frac{\cos \gamma}{I_{M}^{\left(v^{\prime}\right)}}+\frac{1}{M}\left(\cos \gamma_{e}-\cos \gamma\right) \int \Phi_{v^{\prime}}^{2} \frac{\partial^{2} \Phi_{v^{\prime}}}{\partial R_{1} \partial R_{2}} d R_{1} d R_{2},
\end{aligned}
$$

the noncoupling part of the effective potential for bending in the stretching state $v^{\prime}$;

$$
a_{v^{\prime} ; v^{*}} \equiv \frac{1}{2} \int \frac{1}{\mu R_{1}^{2}} \Phi_{v^{\prime}} \Phi_{v^{*}} d R_{1} d R_{2}
$$

the coefficient of nonadiabatic couplings through the centrifugal term;

$$
b_{v^{\prime} ; v^{*}} \equiv \frac{1}{M} \int R_{1}^{-1} R_{2}^{-1} \Phi_{v^{\prime}} \Phi_{v^{*}} d R_{1} d R_{2},
$$

the coefficient of nonadiabatic couplings through momenta;

$$
c_{v^{\prime} ; v^{*}} \equiv b_{v^{\prime} ; v^{*}}+\frac{1}{M} \int R_{2}^{-1} \Phi_{v^{\prime}} \frac{\partial \Phi_{v^{*}}}{\partial R_{1}} d R_{1} d R_{2},
$$

the coefficient of some extra nonadiabatic couplings; and

$$
\begin{aligned}
& w_{v^{\prime} v^{*}}(\gamma) \equiv \int \Phi_{v^{\prime}}\left\{\left[V\left(R_{1}, R_{2}, \gamma_{e}\right)-V\left(R_{1}, R_{2}, \gamma\right)\right] \Phi_{v^{*}}\right. \\
& \left.+\frac{1}{M}\left(\cos \gamma-\cos \gamma_{e}\right) \frac{\partial^{2} \Phi_{v^{*}}}{\partial R_{1} \partial R_{2}}\right\} d R_{1} d R_{2}+b_{v^{\prime} v^{*}} \cos \gamma,
\end{aligned}
$$

nonadiabatic potential couplings. [Note that a factor 2 appears in error on the right-hand side of Eq. (13) in Part $\mathrm{I}^{1}$.]

Decoupling the set of equations (7) by neglecting their right-hand sides leads to the relation

$$
\left[\hat{t}\left(I_{\mu}^{(v)}, I_{M}^{(v)}\right)+W_{v}(\gamma)-E_{v, \tau}^{(\mathrm{ad})}\right] \chi_{v \tau}^{(\mathrm{ad})}=0 .
$$

This equation defines the adiabatic functions $\chi_{v r}^{(\mathrm{ad})}$. Omitting the term $\Delta \hat{t}^{\prime}$ on the right-hand side of Eq. (8c) we obtain an equation for the wave function $\chi_{v r}^{\text {POS }}$ describing motion of two identical particles moving on a sphere of radius $\bar{R}^{(v)}$ around a free heavy particle of mass $M^{(v)}$ :

$$
\left[\hat{t}_{0}\left(I_{\mu}^{(v)}\right)+\Delta \hat{t}\left(I_{M}^{(v)}\right)+W_{v}(\gamma)-E_{v \tau}^{\mathrm{POS}}\right] \chi_{v \tau}^{(\mathrm{POS})}=0,
$$

where the effective masses

$$
m^{(v)}=\left(\frac{1}{\mu}-\frac{1}{M^{(v)}}\right)^{-1}
$$

and $M^{(v)}$ of both the light and heavy particles depend on the degree of stretching excitation. Note that Eq. (15) differs from the Schrödinger equation for particles on a sphere with fixed center ${ }^{7}$ by inclusion of the center of mass correction $\Delta \hat{t}$.

The adiabatic approach discussed here is equivalent to the "adiabatic bend" method of Johnson, Skodje, and Reinhardt, ${ }^{8}$ who treated a bending mode coupled to a single stretch mode. A multidimensional extension of a more sophisticated ap- 
proach, ${ }^{9}$ in which the stretching-mode equation depends parametrically on the bending angle, will be discussed in a future publication. ${ }^{10}$

It should also be noted that a similar decoupling scheme performed using Radau coordinates ${ }^{11-15}$

$$
\begin{aligned}
& \eta_{1}=\alpha_{+} \mathbf{R}_{1}+\alpha \_\mathbf{R}_{2}, \\
& \boldsymbol{\eta}_{2}=\alpha_{-} \mathbf{R}_{1}+\alpha_{+} \mathbf{R}_{2},
\end{aligned}
$$

where

$$
\alpha_{ \pm}=\frac{1}{2}\left(\sqrt{\frac{M}{M+2 m}} \pm 1\right)
$$

leads immediately to a model in which the light particles move on the surface of an immobile sphere in the space spanned by vectors $\eta_{1}$ and $\eta_{2}$. Due to elimination of the center-of-mass correction in Eq. (1), the quantum-mechanical kinetic energy is given simply by the operator $\hat{t}_{0}$. Of course the kinematic separability provided by the Radau coordinates is obtained at the price of a complicated collective motion of the hydrogens, and is most likely associated with large potential energy couplings as a result of mixing between local stretching modes and bending. The effectiveness of employing Radau coordinates for adiabatic separation of motions certainly deserves careful study. ${ }^{16}$

Returning to the set of equations (7), it must be remembered when performing the adiabatic decoupling that the wave functions $\Phi_{n, m,+1}$ and $\Phi_{n, m,-1}$ form a near-degenerate doublet of states for $m>n$, so that couplings between the functions $\chi_{n, m, \kappa, \tau}^{n, m \bar{\kappa}}$ and $\chi_{n, m, \kappa, \tau}^{n, m,-\bar{\kappa}}$ cannot be omitted a priori. These couplings are represented by the operators

$$
\begin{aligned}
\Delta \hat{t}_{n, m}^{\bar{s}_{1}-\bar{\kappa}} \equiv & c_{n, m, \bar{\kappa} ; n, m,-\bar{\kappa}}\left(\hat{\mathbf{R}}_{1} \cdot \nabla_{\theta_{2} \varphi_{2}}-\hat{\mathbf{R}}_{2} \cdot \nabla_{\theta_{1} \varphi_{1}}\right) \\
& +\mathbf{a}_{n, m, \bar{\kappa} ; n, m,-\bar{\kappa}}\left(\Delta_{\theta_{1} \varphi_{1}}-\Delta_{\theta_{2} \varphi_{2}}\right),
\end{aligned}
$$

where

$$
c_{n, m, \bar{\kappa}, n, m,-\bar{\kappa}}=\frac{1}{M} \int R_{2}^{-1} \Phi_{n, m, \bar{\kappa}} \frac{\partial \Phi_{n m-\bar{\kappa}}}{\partial R_{1}} d R_{1} d R_{2},
$$

and the coefficient $a_{n, m, \bar{\kappa}, n, m,-\bar{\kappa}}$ is defined by Eq. (12a). The effects of these couplings, as well as the role of the term omitted in deriving Eq. (15), will be studied in a separate publication.

\section{B. Separation of stretching modes in the two-dimensional equation}

Instead of solving the two-dimensional stretching equation (4) numerically (cf. Ref. 6), we can approximate the appropriate eigenfunctions $\Phi_{m, n, \pm 1}\left(R_{1} R_{2}\right)$ as symmetric and antisymmetric combinations of products of separable stretching functions, i.e., we put

$$
\begin{aligned}
& \Phi_{n, m, \kappa}\left(R_{1} R_{2}\right) \cong \Phi_{n, m}^{[\kappa]}\left(R_{1}, R_{2}\right) \\
& \quad \equiv N_{n m}\left[f_{n}\left(R_{1}\right) f_{m}\left(R_{2}\right)+\kappa f_{m}\left(R_{1}\right) f_{n}\left(R_{2}\right)\right],
\end{aligned}
$$

where

$$
\begin{aligned}
& N_{n m}=1 / \sqrt{2} \text { for } n \neq m, \\
& N_{n m}=1 / 2 \text { for } n=m,
\end{aligned}
$$

and the mode functions are solutions of the one-dimensional equation

$$
\left[-\frac{d^{2}}{d R^{2}}+V\left(R_{1}, R_{e}, \gamma_{e}\right)-\epsilon_{\kappa}\right] f_{\kappa}=0 .
$$

Eigenvalues $E_{n, m, \kappa}^{(2 \mathrm{D})}$ are then approximated by expectation values of the two-dimensional Hamiltonian calculated with functions (21):

$$
\begin{aligned}
E_{n, m, \kappa}^{(2 D)} \cong & \bar{E}_{n, m, \kappa}^{(1)} \equiv \bar{E}_{n, m}^{(1)}+\frac{1}{M} \kappa \cos \gamma_{e}\langle D\rangle_{n m}^{2} \\
& +\int\left[f_{n}^{2}\left(R_{1}\right) f_{m}^{2}\left(R_{2}\right)\right. \\
& \left.+\kappa\left(1-\delta_{n m}\right) f_{n}\left(R_{1}\right) f_{m}\left(R_{1}\right) f_{n}\left(R_{2}\right) f_{m}\left(R_{2}\right)\right] \\
& \times V\left(R_{1}, R_{2}, \gamma_{e}\right) d R_{1} d R_{2}
\end{aligned}
$$

where

$$
\begin{aligned}
& \bar{E}_{n, m}^{(1)} \equiv \epsilon_{n}-\langle V\rangle_{n n}+\epsilon_{m}-\langle V\rangle_{m m}, \\
& \langle D\rangle_{n m} \equiv \int f_{n} \frac{d f_{m}}{d R} d R,
\end{aligned}
$$

and

$$
\langle V\rangle_{n m}=\int f_{n}(R) V\left(R_{1}, R_{e}, \gamma_{e}\right) f_{m}(R) d R .
$$

We find

$$
\begin{aligned}
{\left[R^{(m, n, \kappa)}\right]^{-2} } & \cong\left[\bar{R}^{(n, m)}\right]^{-2} \\
& =\frac{1}{2}\left[\left\langle R^{-2}\right\rangle_{n}+\left\langle R^{-2}\right\rangle_{m}\right] \equiv \mu / \bar{I}_{\mu}^{(n, m)}
\end{aligned}
$$

with

$$
\left\langle R^{-2}\right\rangle_{k} \equiv \int R^{-2} f_{k}^{2}(R) d R=\mu / \bar{I}_{\mu}^{(k)}
$$

and

$$
\begin{aligned}
I_{M}^{(n, m, \kappa)^{-1}} & \cong \bar{I}_{M}^{(n, m, \kappa)^{-1}} \equiv\left\{\bar{M}_{n, m}^{[\kappa]}\left[\bar{R}^{(n, m)}\right]^{2}\right\}^{-1} \\
& \equiv \frac{1}{M}\left[\left\langle R^{-1}\right\rangle_{n n}\left\langle R^{-1}\right\rangle_{m m}+\kappa\left\langle R^{-1}\right\rangle_{n m}^{2}\right]
\end{aligned}
$$

with

$$
\left\langle R^{-1}\right\rangle_{n m} \equiv \int R^{-1} f_{n}(R) f_{m}(R) d R .
$$

The potential (10) takes the form

$$
\begin{aligned}
W_{n, m, \kappa}(\gamma) & \cong \bar{W}_{n, m}^{[\kappa]}(\gamma) \\
& \equiv \bar{W}_{n, m}(\gamma)+\kappa \Delta \bar{W}_{n, m}(\gamma)\left(1-\delta_{n m}\right),
\end{aligned}
$$

where

$$
\begin{aligned}
& \bar{W}_{n, m}(\gamma) \\
& =\bar{E}_{n, m}^{(1)}+\int V\left(R_{1}, R_{2}, \gamma\right) f_{n}^{2}\left(R_{1}\right) f_{m}^{2}\left(R_{2}\right) d R_{1} d R_{2} \\
& \quad-\frac{\cos \gamma}{M\left[\bar{R}^{(n, m)}\right]^{2}}, \\
& \quad \Delta \bar{W}_{n, m}(\gamma) \\
& =\int V\left(R_{1}, R_{2}, \gamma\right) f_{n}\left(R_{1}\right) f_{n}\left(R_{2}\right) f_{m}\left(R_{1}\right) f_{m}\left(R_{2}\right) d R_{1} d R_{2} \\
& +\frac{1}{M} \cos \gamma\langle D\rangle_{n m}^{2} .
\end{aligned}
$$


For $n=m$ we obtain the expressions used in part I to calculate the bending-rotation spectrum of the water molecule in the ground stretching state.

With the approximation (21) for the stretching wave functions, the adiabatic approach leads to the coupled equations for the POS model:

$$
\begin{gathered}
{\left[\hat{t}\left(\bar{I}_{\mu}^{(n, m)}, \bar{I}_{M}^{(n, m, \tilde{\kappa})}\right)+\bar{W}_{n, m}^{[\tilde{\kappa}]}(\gamma)-\bar{E}_{n, m, \tau}^{(\mathbf{a d d})}\right] \chi_{n, m, \kappa, \tau}^{[\tilde{\kappa}]}} \\
=\Delta \hat{t}_{n, m}^{\tilde{\kappa},-\tilde{\kappa}} \chi_{n, m, \kappa, \tau}^{[-\tilde{\kappa}]}, \quad n<m, \quad \tilde{\kappa}= \pm \kappa .
\end{gathered}
$$

We refer to this as the local-mode adiabatic approach. As shown in the next section, this approach can be derived in a more rigorous fashion using an expansion in terms of local mode functions. The POS model corresponds to the case $\bar{\chi}_{n, m, \kappa, \tau}^{[-\kappa]}=0$.

\section{Local-mode expanelon}

We now consider another expansion for the complete vibration-rotation wave function,

$$
\bar{\Psi}_{v, \tau}\left(\mathbf{R}_{1}, \mathbf{R}_{2}\right)=\sum_{n^{\prime}, m^{\prime}, \kappa^{\prime}} \bar{\Phi}_{n^{\prime}, m^{\prime}}^{\left(\kappa^{\prime}\right)}\left(R_{1}, R_{2}\right) \bar{\chi}_{n, m, \kappa_{,}, \mu^{\prime}, \kappa^{\prime}}^{n^{\prime}}\left(\hat{\mathbf{R}}_{1}, \hat{\mathbf{R}}_{2}\right),
$$

which is closely related to the "coupled-bonds" approach recently suggested by two of us. ${ }^{17}$ In fact, transforming Eq. (35) to an equivalent form

$\bar{\Psi}_{v, \tau}\left(\mathbf{R}_{1}, \mathbf{R}_{2}\right)=\sum_{n^{\prime}, m^{\prime}} f_{n^{\prime}}\left(R_{1}\right) f_{m^{\prime}}\left(R_{2}\right) \varphi_{n, m, \kappa_{1} \tau}^{n^{\prime}, m^{\prime}}\left(\hat{R}_{1}, \hat{R}_{2}\right)$,

where

$$
\begin{aligned}
& \varphi_{n, m, \kappa, \tau}^{n^{\prime}, n^{\prime}}=\bar{\chi}_{n, m, \kappa, \tau}^{n^{\prime}, n^{\prime},+1}, \\
& \varphi_{n, m, \kappa, \tau}^{n^{\prime}, m^{\prime}}= \begin{cases}\frac{1}{\sqrt{2}}\left[\bar{\chi}_{n, m, \kappa, \tau}^{n^{\prime}, m^{\prime},+1}+\bar{\chi}_{n, m, \kappa, \tau}^{m^{\prime}, n^{\prime}, \tau^{-1}}\right], & n^{\prime}<m^{\prime}, \\
\frac{1}{\sqrt{2}}\left[\bar{\chi}_{n, m, m_{, \tau}}^{n^{\prime}, m^{\prime},+1}-\bar{\chi}_{n, m, \kappa, \tau}^{m^{\prime}, n^{\prime}, \tau^{-1}}\right], & n^{\prime}>m^{\prime},\end{cases}
\end{aligned}
$$

and expanding the functions $\varphi_{,, m, \kappa, \tau}^{n^{\prime}, m^{\prime}}$ in terms of spherical harmonics $Y_{m_{1}}^{j_{1}}\left(\widehat{\mathbf{R}}_{1}\right)$ and $Y_{m_{2}}^{j_{2}}\left(\hat{\mathbf{R}}_{2}\right)$ as done in $I$, we obtain an expansion ${ }^{17}$ in which each bond is treated as a nearly rigid rotor.

The set of coupled equations for the functions $\bar{\chi}_{n, m, x^{\prime}}^{n^{\prime}, m^{\prime}, \kappa^{\prime}}$ namely:

$$
\begin{aligned}
& {\left[\hat{t}\left(\bar{I}_{\mu}^{(n, m)}, \bar{I}_{m}^{(n, m, \kappa)}\right)+\bar{W}_{n, m}^{(\kappa)}(\gamma)-\bar{E}_{n, m, \kappa, \tau}\right] \bar{\chi}_{n, m, \kappa}^{n, m, \kappa}} \\
& =\sum_{v^{\prime} \neq v}\left[\bar{a}_{v, v^{\prime}}\left(\Delta_{\theta_{1} \varphi_{1}}+\kappa \kappa^{\prime} \Delta_{\theta_{2} \varphi_{2}}\right)+\bar{b}_{v, v^{\prime}} \nabla_{\theta_{1} \Phi_{1}} \cdot \nabla_{\theta_{2} \varphi_{2}}\right. \\
& \left.+\bar{c}_{v, v^{\prime}}\left(\hat{\mathbf{R}}_{1} \cdot \nabla_{\theta_{2} \Phi_{2}}+\kappa \kappa^{\prime} \hat{\mathbf{R}}_{2} \cdot \nabla_{\theta_{1} \varphi_{1}}\right)-\bar{W}_{v, v^{\prime}}(\gamma)\right] \bar{\chi}_{n, m, \kappa}^{n^{\prime}, m^{\prime}, \kappa^{\prime}},
\end{aligned}
$$

is clearly similar to Eq. (7). In Eq. (38), the coefficients $a, b$, and $c$ can be obtained by substituting Eq. (21) into Eqs. (12a), (12b), and (12c), respectively. The quantities $\bar{M}^{[\kappa]}$ and $\overline{\boldsymbol{R}}^{(n, m)}$ and the potential $\bar{W}_{n, m}^{[\kappa]}(\gamma)$ have already been defined in Eqs. (28), (30), and (32). The only new coefficient is

$$
\begin{aligned}
\bar{W}_{v, v^{\prime}} \equiv & \bar{W}_{m, n, \kappa ; m^{\prime}, n^{\prime}, \kappa^{\prime}} \\
= & \delta_{\kappa \kappa^{\prime}}\left\{\int \Phi_{m^{\prime} n^{\prime}}^{\left[\kappa^{\prime}\right]} \Phi_{m n}^{[\kappa]} V\left(R_{1}, R_{2}, \gamma\right) d R_{1}, d R_{2}\right. \\
& -\langle V\rangle_{n n^{\prime}} \delta_{m m^{\prime}}-\langle V\rangle_{m m^{\prime}} \delta_{n n^{\prime}} \\
& +N_{n m}^{n^{\prime} m^{\prime}} \frac{\cos \gamma}{M}\left[\langle D\rangle_{n^{\prime} n}\langle D\rangle_{m^{\prime} m}\right. \\
& \left.\left.+\kappa\langle D\rangle_{n^{\prime} m}\langle D\rangle_{m^{\prime} n}\right]\right\},
\end{aligned}
$$

where

$$
N_{n m}^{n^{\prime} m^{\prime}} \equiv \begin{cases}1, & n^{\prime} \neq m^{\prime}, \quad n \neq m ; \\ N_{n m}, & n^{\prime}=m^{\prime} ; \\ N_{n^{\prime} m^{\prime}}, & n=m .\end{cases}
$$

It is crucial to note that an adiabatic approximation applied to the set of equations (38) yields precisely the same equations as those obtained by applying the separability approximation (21) to the 2D-stretch adiabatic approach. As long as all terms in Eq. (34) are kept, the same adiabatic approximation results as obtained starting from the expansion (36).

We now look for solutions of Eq. (34) which are adiabatic approximations to the expansion functions $\varphi$ in Eq. (36). Thus, we set

$$
\begin{aligned}
& \varphi_{n, m, \kappa, \tau} \equiv 2^{-1 / 2}\left[\bar{\chi}_{n, m, \kappa, \tau}^{+}+\bar{\chi}_{n, m, \kappa, \tau}^{-}\right], \\
& \varphi_{m, n, \kappa, \tau} \equiv 2^{-1 / 2}\left[\bar{\chi}_{n, m, \kappa, \tau}^{+}-\bar{\chi}_{n, m, \kappa, \tau}^{-}\right]
\end{aligned}
$$

with $n<m$. Adding and subtracting equations (34) corresponding to opposite values of $\tilde{\kappa}$ and to a fixed value of $\kappa$ we obtain an equivalent pair of equations:

$$
\begin{aligned}
& {\left[\hat{t}\left(\bar{I}_{\mu}^{(n)}, \bar{I}_{\mu}^{(m)}, \bar{I}_{n, m}\right)+\bar{W}_{n, m}(\gamma)-\bar{E}_{n, m, \kappa, \tau}^{(\text {ad) }}\right] \varphi_{k, l, \kappa, \tau}} \\
& +\left[\hat{t}_{1}\left(M\left\langle R^{-1}\right\rangle_{n m}^{-2}\right)+\Delta W_{n, m}(\gamma)\right. \\
& \left.-c_{n, m, 1 ; n, m,-1}\left(\hat{\mathbf{R}}_{1} \cdot \nabla_{\theta_{2} \varphi_{2}}-\hat{\mathbf{R}}_{2} \cdot \nabla_{\theta_{1} \varphi_{1}}\right)\right] \varphi_{k, l, \kappa, \tau}=0,
\end{aligned}
$$

where

$$
\begin{aligned}
& \hat{t}\left(\bar{I}_{1}, \bar{I}_{2}, \bar{I}\right)=\hat{t}_{0}\left(\bar{I}_{1}, \bar{I}_{2}\right)+\hat{t}_{1}(\bar{I}), \\
& \hat{t}_{0}\left(\bar{I}_{1}, \bar{I}_{2}\right) \equiv-\frac{1}{2 \bar{I}_{1}} \Delta_{\theta_{1} \Phi_{1}}-\frac{1}{2 \bar{I}_{2}} \Delta_{\theta_{2} \Phi_{2}}, \\
& \bar{I}_{n, m} \equiv M\left[\left\langle R^{-1}\right\rangle_{n n}\left\langle R^{-1}\right\rangle_{m m}\right]^{-1}, \\
& \bar{M}_{n, m} \equiv \bar{I}_{n, m} /\left(\bar{R}^{(n)} \bar{R}^{(m)} .\right.
\end{aligned}
$$

In deriving, Eq. (42) we have also taken into account the fact that

and

$$
\begin{aligned}
a_{n, m, \bar{\kappa}, n, m,-\bar{\kappa}} & =a_{n, m,-\bar{\kappa} ; n, m, \bar{\kappa}} \\
& =\frac{1}{4 \mu}\left[\left\langle R^{-2}\right\rangle_{n}-\left\langle R^{-2}\right\rangle_{m}\right]
\end{aligned}
$$

$$
c_{n, m, \bar{\kappa} ; n, m,-\bar{\kappa}}=-c_{n, m,-\bar{\kappa}, n, m, \bar{\kappa}} .
$$

Decoupling the new set of equations (42) gives an equation for two particles moving on concentric spheres of radii $\bar{R}^{(k)}$ and $\bar{R}^{(l)}$ with a freely moving center:

$$
\begin{aligned}
& {\left[\hat{t}_{0}\left(\bar{I}_{\mu}^{(n)}, \bar{I}_{\mu}^{(m)}\right)+\Delta \hat{t}\left(\bar{I}_{n, m}\right)+\bar{W}_{n, m}(\gamma)-\bar{E}_{n, m, \kappa}^{\text {(POCS) }}\right]} \\
& \quad \times \varphi_{k, l, \kappa}^{\text {(PoCs) }}=0 \\
& \quad k=m, l=n \text { or } k=n, l=m .
\end{aligned}
$$


Comparison with the model in which the two particles move on concentric spheres with a fixed center, ${ }^{18}$ we see that an additional center-of-mass correction $\Delta \hat{t}$ has been introduced here. Calculation of the matrix elements of this term in a basis of spherical harmonics is done as for the case of two particles on the same sphere, ${ }^{1}$ and is not discussed further here.

It should be noted that the function:

$$
\chi_{n, m, \tau,+1}^{\text {(POCS) }} \equiv 2^{-1 / 2}\left(\varphi_{n, m, \tau}^{\text {(POCS) }}+\varphi_{m, n, \tau}^{\text {(POCS) }}\right)
$$

describes motion of two particles on a sphere of radius $\bar{R}^{(n, m)}$ interacting with the potential $\bar{W}_{n, m}(\gamma)$. This implies that for $n<m$ the function $\chi_{n, m, \tau,+1}^{(\mathrm{POCS})}$ should closely resemble the functions $\bar{\chi}_{n, m, \pm 1, \tau}^{\text {(POS) }}$ of the POS model, since the three potentials $\bar{W}_{n, m}^{+}(\gamma), \bar{W}_{n, m}^{-}(\gamma)$, and $\bar{W}_{n, m}(\gamma)$ and three effective masses $\bar{M}_{n, m}^{+}, \bar{M}_{n, m}^{-}$, and $\bar{M}_{n, m}$ become very similar in this limit. The two models must therefore predict similar values for bending frequencies and rotational structure in highly excited local mode states. The calculations shown below support this conclusion.

Note that the above discussion refers only to energy levels and spacings, not to intensities. The total wave functions given by the two models are essentially different. In fact, we have

$$
\Psi_{n, m, \kappa, \tau}^{(\mathrm{POS})}\left(\mathbf{R}_{1}, \mathbf{R}_{2}\right)=\Phi_{n, m}^{[\kappa]}\left(R_{1}, R_{2}\right) \chi_{n, m, \kappa, \tau}^{(\mathrm{POS})}\left(\widehat{\mathbf{R}}_{1}, \widehat{\mathbf{R}}_{2}\right)
$$

and

$$
\begin{aligned}
\Psi_{n, m, \kappa, \tau}^{(\text {POCS }}\left(\mathbf{R}_{1}, \mathbf{R}_{2}\right)= & \Phi_{n, m}^{+}\left(R_{1}, R_{2}\right) \chi_{n, m, \tau, \kappa}^{(\text {POCS })}\left(\hat{\mathbf{R}}_{1}, \widehat{\mathbf{R}}_{2}\right) \\
& +\Phi_{n, m}^{-}\left(R_{1}, R_{2}\right) \chi_{n, m, \tau,-\kappa}^{(\text {POCS })}\left(\widehat{\mathbf{R}}_{1}, \hat{\mathbf{R}}_{2}\right)
\end{aligned}
$$

where the function

$$
\chi_{n, m, \tau_{2}-1}^{\text {(POCS) }} \equiv 2^{-1 / 2}\left(\varphi_{n, m, \tau}^{\text {(POCS) }}-\varphi_{m, n, \tau}^{\text {(POCS })}\right.
$$

is not expected to vanish.

TABLE 1. Radii of the spheres and effective masses of the oxygen calculated with the Sorbie-Murrell potential as modified by Lawton and Child (Ref. 6). All the values are in atomic units.

\begin{tabular}{ccccccc}
\hline \hline$n+m$ & $n m^{\alpha}$ & $\bar{R}^{(n, m)}$ & $\bar{R}^{(m)}$ & $\bar{R}^{(n)}$ & $\bar{M}_{n, m}^{(\kappa)}$ & $\bar{M}_{n, m}$ \\
\hline 1 & $01^{+}$ & 0.9753 & 0.9846 & 0.9662 & 16.080 & \\
& $01^{-}$ & & & & 16.238 & 16.156 \\
2 & $02^{+}$ & & & & 16.245 & \\
& $02^{-}$ & 0.9845 & 1.0039 & 0.9662 & 16.252 & 16.237 \\
& $11^{+}$ & 0.9846 & & & 16.236 & 16.236 \\
3 & $03^{+}$ & & & & 16.346 & \\
& $03^{-}$ & 0.9940 & 1.0244 & 0.9662 & 16.347 & 16.319 \\
& $12^{+}$ & & & & 16.165 & \\
& $12^{-}$ & 0.9942 & 1.0039 & 0.9846 & 16.480 & 16.318 \\
4 & $04^{+}$ & & & & 16.454 & \\
& $04^{-}$ & 1.0038 & 1.0463 & 0.9662 & 16.454 & 16.402 \\
& $13^{+}$ & & & & 16.403 & \\
& $13^{-}$ & 1.0039 & 1.0244 & 0.9846 & 16.424 & 16.400 \\
& 22 & 1.0039 & & & 16.400 & 16.400 \\
5 & $05^{+}$ & & & & 16.573 & \\
& $05^{-}$ & 1.0142 & 1.0701 & 0.9662 & 16.573 & 16.487 \\
& $14^{+}$ & & & & 16.513 & 16.484 \\
& $14^{-}$ & 1.0140 & 1.0463 & 0.9846 & 16.516 & 16.484 \\
& $23^{+}$ & & & & 16.255 & 16.483 \\
& $23^{-}$ & 1.0140 & 1.0244 & 1.0039 & 16.724 & \\
\hline \hline
\end{tabular}

The POS model is certainly convenient since it enables a significantly smaller basis to be used when the symmetry of the problem is exploited. However, it is not clear $a$ priori which of the models gives better results for transition intensities. The POCS model is more general, since it can immediately be extended to XYZ molecules such as the HOD isotopic modification of water. In fact, Eq. (48) can be interpreted either as describing motion of two particles of different mass on a single sphere, or on two different spheres, depending on how the particle masses are defined. The outstanding question is whether it is possible to give the spheres more physical significance by invoking the Franck-Condon principle.

\section{RESULTS}

We tested both the POS and POCS models on excited stretching states of the water molecule using the Sorbie-Murrell potential as modified by Lawton and Child. ${ }^{6}$ Our choice of potential was determined by the fact that Lawton and Child ${ }^{6,19}$ studied both the two- and three-dimensional vibrational problem, so that it is possible to estimate errors resulting from the use of local-mode wave functions (21) instead of solutions $\Phi_{n, m, \kappa}\left(R_{1}, R_{2}\right)$ of the two-dimensional problem.

It would also be of interest to attempt to reproduce the results of the Reimers-Watts calculations ${ }^{5}$ using our model. Unfortunately this was not possible. Reimers and Watts ${ }^{5} \mathrm{ex}$ press the Wilson-Howard-Watson Hamiltonian ${ }^{20,21}$ in terms of rectilinear vibrational coordinates, which are not precisely defined in their paper. Our guess is that these authors have exploited a set of rectilinear coordinates "locally coincident" (in the terminology of Ref. 22) with the geometrical internal coordinates defined in their equation (24); however, in this case, their potential is mass dependent and should not be used for study of isotopic modifications of water as done by Reimers and Watts. ${ }^{5}$ Note that the main difficulty which Reimers and Watts try to overcome is that the Wilson-Howard-Watson Hamiltonian is inappropriate for molecules exhibiting localmode behavior, in contrast to the method presented here.

In Table I we present calculated sphere radii and effective central (oxygen) atom masses for stretching states with no more than five quanta of excitation. It is an intriguing result that the effective masses of the oxygen atom in the POCS model and the sphere radii in the POS model depend only on the total number of stretching quanta.

It should be noted that the effective mass $\bar{M}_{0}$ in Table III of part I was calculated by means of the formula:

$$
\bar{M}_{0}=\bar{I}_{00} / R_{e}^{2},
$$

TABLE II. Effective masses (a.u.) of the oxygen in the water molecule and its $C_{2 v}$ isotopic modifications.

\begin{tabular}{lccc}
\hline \hline & \multicolumn{3}{c}{$\bar{M}_{\text {o0 }}$ (a.u.) } \\
\cline { 2 - 4 } & $\mathrm{CCL}$ & $\mathrm{B}(A)$ & $\mathrm{B}(B)$ \\
\hline $\mathrm{H}_{2} \mathrm{O}$ & 16.077 & 16.076 & 16.075 \\
$\mathrm{D}_{2} \mathrm{O}$ & 16.053 & 16.052 & 16.051 \\
$\mathrm{~T}_{2} \mathrm{O}$ & 16.045 & 16.045 & 16.043 \\
\hline
\end{tabular}


TABLE III. Radii of the spheres and effective masses of the oxygen calculated with Botschwina's (Ref. 27) potential B. All the values are in atomic units.

\begin{tabular}{ccccccc}
\hline \hline$n+m$ & $n m^{\kappa}$ & $\bar{R}^{(n, m)}$ & $\bar{R}^{(m)}$ & $\bar{R}^{(n)}$ & $\bar{M}_{n, m}^{[\kappa]}$ & $\bar{M}_{n, m}$ \\
\hline 1 & $01^{+}$ & \multirow{2}{*}{0.9717} & 0.9784 & 0.9651 & 16.075 & 16.233 \\
& $01^{-}$ & & & & 16.153 \\
2 & $02^{+}$ & & & & \\
2 & $02^{-}$ & 0.9754 & 0.9861 & 0.9651 & 16.228 & \\
& $11^{+}$ & 0.9784 & & & 16.233 & 16.227 \\
& & & & & \\
\hline \hline
\end{tabular}

where $R_{e}$ is the equilibrium bond length. Calculation of the mass $\bar{M}_{0}$ using Eq. (45b) shows that it is essentially indepen. dent of the choice of potential surface. Recalculated values of the oxygen effective mass in the water molecule and its $C_{2 v}$ isotopic modifications are shown in Table II for the CarneyCurtiss-Langhoff ${ }^{23}$ (CCL) and two Botschwina ${ }^{24}$ (B) potentials. Using the Lawton-Child potential gives the value $\bar{M}_{00}=16.075$ a.u.

Excitation of stretching modes makes both quantities $\bar{R}^{(n, m)}$ and $\bar{M}_{n, m}$ more sensitive to the choice of potential surface, as can be seen by comparing the first lines of Table I with Table III, which was obtained using Botschwina's potential B. The scatter of values within the multiplet $m+n=2$ is also larger.

In Table IV we use the notation $\Delta v_{2 D}^{(v)}$ for the difference

$$
\Delta v_{2 D}^{(\nu)}=\left(\bar{E}_{n, m, \kappa}^{(1)}-\bar{E}_{0,0}^{(1)}\right)-v_{n, m, \kappa}^{(2 D)},
$$

where values $v_{n, m, \kappa}^{(2 D)} \equiv \bar{E}_{n, m, \kappa}^{(2 D)}-\bar{E}_{00,+1}^{(2 d)}$ are taken from the paper by Lawton and Child. ${ }^{6}$ The second column of Table IV shows differences between vibrational frequencies calculated by means of POS model and those calculated by Child and Lawton ${ }^{19}$ using the accurate Hamiltonian. It is remarkable that
TABLE IV. Errors in calculating vibrational frequencies by means of the POS model based on the local-mode expansion. The differences $\Delta v_{2 D}^{(v)}$ are determined by relation (52), where the eigenvalues of the two-dimensional problem were taken from Ref. 6 . The notation $\Delta v_{3 D}^{(v)}$ is used for the differences between the POS predictions for vibrational frequencies and the values calculated by Child and Lawton (Ref. 19) using the accurate Hamiltonian. Here and below all the frequencies are given in $\mathrm{cm}^{-1}$.

\begin{tabular}{lllrrr}
\hline$v_{1}$ & $v_{3}$ & $n m^{[\kappa]}$ & $\Delta v_{2 D}^{(0)}$ & \multicolumn{1}{c}{$\Delta v_{3 D}^{(v)}$} & $\Delta v_{3 \mathrm{D}}^{(0)}-\Delta v_{2 D}^{(v)}$ \\
\hline 1 & 0 & $01^{+}$ & -7 & -7 & 0 \\
0 & 1 & $01^{-}$ & -6 & -10 & -4 \\
2 & 0 & $02^{+}$ & 42 & 34 & -8 \\
1 & 1 & $02^{-}$ & -12 & -16 & -4 \\
0 & 2 & $11^{+}$ & -68 & -60 & 8 \\
1 & 2 & $12^{+}$ & -58 & -47 & 11 \\
0 & 3 & $12^{-}$ & -38 & -39 & -1 \\
4 & 0 & $13^{+}$ & 82 & 64 & -18 \\
1 & 3 & $13^{-}$ & -48 & -43 & 5 \\
1 & 4 & $23^{+}$ & -141 & -101 & 40 \\
0 & 5 & $23^{-}$ & -76 & -68 & 8 \\
0 & 4 & $22^{+}$ & -159 & -120 & 39 \\
3 & 0 & $03^{+}$ & 22 & 10 & \\
2 & 1 & $03^{-}$ & 3 & -5 & \\
2 & 2 & $04^{+}$ & 9 & -6 & \\
3 & 1 & $04^{-}$ & 5 & -9 & \\
3 & 2 & $05^{+}$ & 0.6 & -24 & \\
4 & 1 & $05^{-}$ & 0.0 & -24 & \\
5 & 0 & $14^{+}$ & 61 & 30 & \\
2 & 3 & $14^{-}$ & -5 & -16 & \\
\hline \hline
\end{tabular}

for states with $m-n<3$ most of the error is caused by use of the local-mode expansion instead of the two-dimensional stretching approach. That is, interaction between stretching modes is important in these states. As expected, the difference between the two approaches decreases upon increasing the number of quanta in one bond compared to the other.

Note that the splitting $E_{n, m,+1}^{(1)}-E_{n, m,-1}^{(1)}$ is determined by the expression:

$\bar{E}_{n, m,+1}^{(1)}-\bar{E}_{n, m,-1}^{(1)}=2 \int f_{n}\left(R_{1}\right) f_{m}\left(R_{1}\right) f_{n}\left(R_{2}\right) f_{m}\left(R_{2}\right) V\left(R_{1}, R_{2}, \gamma_{e}\right) d R, d R_{2}+\frac{2}{M} \cos \gamma_{e}\langle D\rangle_{n m}^{2}$.

For $n=0, m=1$, relation (54) gives the value $106.5 \mathrm{~cm}^{-1}$, whereas the numerical solution of Eq. (4) by Lawton and Child ${ }^{6}$ gives $106.3 \mathrm{~cm}^{-1}$. Using the POS model decreases the splitting to $98.9 \mathrm{~cm}^{-1}$. This decrease is only in qualitative agreement with the calculations of Child and Lawton, ${ }^{19}$ who obtained the value $102.1 \mathrm{~cm}^{-1}$ using the accurate Hamiltonian. Exciting the bending mode results in a slight increase in the splitting ( $104.2 \mathrm{~cm}^{-1}$ for $v_{2}=1,107.7 \mathrm{~cm}^{-1}$ for $\left.v_{2}=2\right)$ in qualitative agreement with Ref. $19\left(103.7 \mathrm{~cm}^{-1}\right.$ for $v_{2}=1$, $104.9 \mathrm{~cm}^{-1}$ for $v_{2}=2$ ).

As can be seen from Table V, relation (54) gives the major contribution to the splitting for other states with $m-n=1$, although agreement is worse. For states with one mode highly excited neglect of interactions between bonds become a questionable approximation for the calculation of the splittings.

Since the POCS model does not resolve doublets $\mathrm{nm}^{+}$and $\mathrm{nm}^{-}$, it is clearly not suitable for describing states with $m-n=1$ for which splittings are relatively large. However, for states with an excess of at least two quanta in one bond the accuracy of both the POS and POCS models becomes of the same order. For example, the POS model gives $G(2,0,0)=7239.6 \mathrm{~cm}^{-1}$ and $G(1,0,1)=7241.0 \mathrm{~cm}^{-1}$, while both levels are represented in the POCS model by the single number $7240.0 \mathrm{~cm}^{-1}$.

Note that the correspondence between local and normal mode assignments used here follows the work of Child and Lawton. ${ }^{19}$ As pointed out by these authors, the $\left(4 v_{2} 0\right)$ levels are in fact higher in energy than the $\left(2 v_{2} 2\right)$ levels and the $\left(5 v_{2} 0\right)$ levels higher than the $\left(3 v_{2} 2\right)$ levels due to anharmonic coupling. This is in contrast to the assignments made on the basis of their two-dimensional treatment of the water molecule. Reimers and Watts apparently overlooked the more recent 
TABLE V. Splittings of vibrational levels in doublets of states having the same number of quanta in one bond.

\begin{tabular}{|c|c|c|c|c|}
\hline \multirow[b]{2}{*}{$n, m$} & \multicolumn{4}{|c|}{$G_{n, m-1}-G_{n, m,+1}$} \\
\hline & [6] & Ref. 53 & [19] & POS \\
\hline 01 & 106.3 & 106.5 & 102.1 & 98.8 \\
\hline 12 & 201.2 & 220.8 & 176.6 & 185.2 \\
\hline 23 & 277.6 & 342.7 & 224.1 & 256.6 \\
\hline 02 & 57.6 & 3.3 & 51.0 & 1.4 \\
\hline 13 & 138.2 & 8.3 & 109.1 & 2.4 \\
\hline 03 & 19.8 & $\ldots$ & 15.0 & ... \\
\hline 14 & 67.2 & 0.8 & 43.8 & 1.8 \\
\hline
\end{tabular}

work of Child and Lawton, and so have incorrectly assigned the levels (400s), (310s), (401s), (500s), (410s) [in the notation of Ref. 5; these are normal mode states (202), (400), (212), (302), (500) ]. Experimental values are available for all these levels (Ref. 17), and are in reasonable agreement with the values calculated by Reimers and Watts ${ }^{5}$ in view of the simple form of their potential.

In Table VI we study the effect of excitation of stretching modes on the bending frequencies. Since the calculated value of $G(010)$ is not given in Ref. 19, we have given in Table VI the shifts of bending frequencies relative to that in the state (001). As a rule the POS tends of overestimate these splittings.

It should be cautioned that the good agreement seen in Table VI is probably a fortuitous consequence of the choice of potential. In particular, use of the POS model with Botsch-

TABLE VI. Changes of the bending frequencies due to excitation of stretching modes in the accurate calculations (Ref. 19) and our model calculations.

\begin{tabular}{|c|c|c|c|c|c|}
\hline \multirow[b]{3}{*}{$v_{1} v_{3}$} & \multicolumn{5}{|c|}{$G\left(v_{1}, 0, v_{3}\right)-G\left(v_{1}, v_{2}, v_{3}\right)-G(0,0,1)+G\left(0, v_{2}, 1\right)$} \\
\hline & \multirow[b]{2}{*}{$n m^{[\kappa]}$} & \multicolumn{2}{|c|}{$v_{2}=1$} & \multicolumn{2}{|c|}{$\quad v_{2}=2$} \\
\hline & & POS & [19] & POS & [19] \\
\hline 10 & $01^{+}$ & 5 & 2 & 9 & 3 \\
\hline 02 & $11^{+}$ & 30 & 27 & 56 & 54 \\
\hline 20 & $\mathrm{O2}^{+}$ & 31 & 32 & 59 & 64 \\
\hline 11 & $\mathbf{0 2}-$ & 33 & 32 & 61 & 65 \\
\hline 03 & $12^{-}$ & 54 & 59 & 103 & 115 \\
\hline 12 & $12^{+}$ & 68 & 67 & 130 & 131 \\
\hline 30 & $\mathbf{0 3}^{+}$ & 68 & 71 & 129 & 138 \\
\hline 21 & $\mathrm{O3}^{-}$ & 68 & 72 & 129 & 141 \\
\hline 04 & $22^{+}$ & 95 & 94 & 183 & 186 \\
\hline 40 & $13^{+}$ & 96 & 108 & 185 & 212 \\
\hline 13 & $13^{-}$ & 100 & 106 & 193 & 208 \\
\hline 22 & $04^{+}$ & 107 & 114 & 207 & 224 \\
\hline 31 & $04^{-}$ & 107 & 115 & 207 & 226 \\
\hline 05 & $23^{-}$ & 121 & 134 & 235 & 265 \\
\hline 14 & $23^{+}$ & 144 & 148 & 278 & 289 \\
\hline 50 & $14^{+}$ & 139 & 153 & 270 & 299 \\
\hline 23 & $14^{-}$ & 140 & 154 & 271 & 301 \\
\hline 32 & $05^{+}$ & 152 & 159 & 296 & 319 \\
\hline 41 & $05^{-}$ & 152 & 159 & 296 & 320 \\
\hline
\end{tabular}

TABLE VII. Changes of the bending frequencies due to giving two quanta to excitation of stretching modes, according to calculations performed with Botschwina's potential B.

\begin{tabular}{|c|c|c|c|c|c|c|c|}
\hline \multirow[b]{3}{*}{$v_{1} v_{3}$} & \multicolumn{6}{|c|}{$G\left(v_{1}, 0, v_{3}\right)-G\left(v_{1}, v_{2}, v_{3}\right)+G\left(0, v_{2}, 1\right)-G(0,0,1)$} & \\
\hline & \multirow[b]{2}{*}{$n m^{[\kappa]}$} & \multicolumn{2}{|c|}{$v_{2}=1$} & \multicolumn{2}{|c|}{$v_{2}=2$} & $v_{2}=3$ & \\
\hline & & ours & $\mathbf{B}(\boldsymbol{B})$ & ours & $\mathbf{B}(\boldsymbol{B})$ & ours & $\mathbf{B}(B)$ \\
\hline 02 & $11^{+}$ & 18 & 16 & 45 & 33 & 52 & 47 \\
\hline 11 & $02^{-}$ & 12 & 20 & 24 & 44 & 36 & 67 \\
\hline
\end{tabular}

wina's potential $B$ does not reveal any regularities in the discrepancies between exact and predicted shifts (see Table VII).

Analysis of experimental data ${ }^{25-33}$ shows that excitation of stretching modes leads to changes in rotational levels that are of the order of the absolute errors in the calculations performed both here and in Ref. 5 . In view of this, we do not find Table 4 of Ref. 5 convincing. As can be seen from Tables VIII and IX of the present paper, the potential suggested by Reimers and Watts ${ }^{5}$ is not sufficiently accurate to describe the observed pattern of rotational structure.

In Tables VIII-X we present energy differences obtained by subtracting rotational energies in excited vibrational states $\left(v_{1} v_{2} v_{3}\right)$ from those in the state $\left(0 v_{2} 0\right)$, i.e., no excitation in stretching modes. For the $\mathrm{O1}^{+}$and $01^{-}$states there is relatively good agreement between observed $\mathrm{d}^{25-27,29,30}$ and $P O S$ values except for $3_{31}$ and $3_{30}$ rotational levels in the second and third excited bending states. The POS model also predicts the correct sign for rotational level shifts due to excitation of two different one-quantum stretching levels, but underestimates the magnitudes. The POS model also provides reasonably accurate predictions of changes in rotational structure due to the presence of two quanta in two local modes, even though it does not lead to any distinction between shifts for the three vibrational states of $02^{+}, \mathrm{O2}^{-}$, and $11^{+}$(in contrast to experiment, Ref. 28). Theoretical values presented in Table IX are for the $02^{-}$or $\left(1 v_{2} 1\right)$ state. The results of Reimers and Watts for the other two vibrational states are very close, whereas the POS model leads to essentially the same rotational energies in all three states.

When discussing the rotational structure for $\mathrm{H}_{2} \mathrm{O}$ in the $02^{-}, 03^{-}$, and $12^{-}$states Reimers and Watts ${ }^{5}$ refer to the old experimental data of Mecke and collaborators. ${ }^{32}$ Comparison with the data of Toth and Margolis ${ }^{28}$ for the $0^{-}$state shows that the older measurements were not sufficiently accurate. The data of Ref. 35 for $03^{-}$and $12^{-}$should also be viewed with suspicion because they do not provide a monotonic decrease of rotational energies with increasing numbers of quanta in local modes.

For states with five quanta in local modes even experimental rotational structures ${ }^{33}$ become very close, at least for the states with all five quanta in one bond, ${ }^{34}$ which are presented in Table X. However as one can see from Table X, rotational energies in the rotational states $3_{31}$ and $3_{30}$ turn out to be significantly overestimated.

It should finally be mentioned that, starting with a total of two quanta of local mode excitation, the POS and POCS models give rise to very similar rotational structures. 
TABLE VIII. Changes in rotational levels due to excitation of stretching modes. Tables VIII-X give differences between the rotational energies of states $\left(\mathrm{Ov}_{2} \mathrm{O}\right)$ with no excitation in stretching modes and the energies of the same rotational states of various levels $\left(v_{1} v_{2} v_{3}\right)$ with the same number of quanta in the bending mode and various numbers $v_{1}$ and $v_{3}$ of quanta in the symmetric and antisymmetric stretching modes, respectively. This table deals with one quantum of stretching excitation and (a) no bending excitation, (b) one quantum of bending excitation, (c) two quanta of bending excitation, and (d) three quanta of bending excitation.

\begin{tabular}{|c|c|c|c|c|c|c|c|c|c|c|c|}
\hline \multirow{2}{*}{$\begin{array}{l}\overline{\text { (a) }} \\
J\end{array}$} & \multirow[b]{2}{*}{$\boldsymbol{K}_{a}$} & \multirow[b]{2}{*}{$\boldsymbol{K}_{\mathrm{c}}$} & \multicolumn{3}{|c|}{$(100)$} & \multicolumn{3}{|c|}{$(001)$} & \multicolumn{3}{|c|}{$v^{+}-v^{-}$} \\
\hline & & & Expt & POS & Ref. 5 & Expt & POS & Ref. 5 & Expt & POS & Ref. 5 \\
\hline 1 & $\begin{array}{l}0 \\
1 \\
1\end{array}$ & $\begin{array}{l}1 \\
1 \\
0\end{array}$ & $\begin{array}{l}0.4 \\
0.9 \\
1.0\end{array}$ & $\begin{array}{l}0.4 \\
1.0 \\
1.0\end{array}$ & $\begin{array}{l}0.4 \\
0.6 \\
0.7\end{array}$ & $\begin{array}{l}0.2 \\
1.3 \\
1.3\end{array}$ & $\begin{array}{l}0.3 \\
1.2 \\
1.1\end{array}$ & $\begin{array}{l}0.2 \\
0.6 \\
0.8\end{array}$ & $\begin{array}{r}0.2 \\
-0.4 \\
-0.4\end{array}$ & $\begin{array}{r}0.1 \\
-0.2 \\
-0.1\end{array}$ & $\begin{array}{r}-0.2 \\
0.0 \\
-0.1\end{array}$ \\
\hline 2 & $\begin{array}{l}0 \\
1 \\
1 \\
2 \\
2\end{array}$ & $\begin{array}{l}2 \\
2 \\
1 \\
1 \\
0\end{array}$ & $\begin{array}{l}1.2 \\
1.7 \\
1.8 \\
3.3 \\
3.3\end{array}$ & $\begin{array}{l}1.1 \\
1.7 \\
1.7 \\
3.9 \\
3.7\end{array}$ & $\begin{array}{l}1.3 \\
1.5 \\
1.6 \\
2.2 \\
1.7\end{array}$ & $\begin{array}{l}0.8 \\
1.9 \\
1.7 \\
5.1 \\
5.0\end{array}$ & $\begin{array}{l}0.9 \\
1.8 \\
1.6 \\
4.6 \\
4.4\end{array}$ & $\begin{array}{l}0.7 \\
1.2 \\
1.1 \\
2.5 \\
2.5\end{array}$ & $\begin{array}{r}0.4 \\
-0.2 \\
0.1 \\
-1.8 \\
-1.7\end{array}$ & $\begin{array}{r}0.2 \\
-0.1 \\
0.1 \\
-0.7 \\
-0.7\end{array}$ & $\begin{array}{r}0.6 \\
0.3 \\
0.5 \\
-0.3 \\
-0.8\end{array}$ \\
\hline 3 & $\begin{array}{l}0 \\
1 \\
1 \\
2 \\
2 \\
3 \\
3\end{array}$ & $\begin{array}{l}3 \\
3 \\
2 \\
2 \\
1 \\
1 \\
0\end{array}$ & $\begin{array}{l}2.5 \\
2.8 \\
3.1 \\
4.5 \\
4.5 \\
7.0 \\
7.1\end{array}$ & $\begin{array}{l}2.4 \\
2.8 \\
2.9 \\
4.9 \\
4.7 \\
8.5 \\
8.5\end{array}$ & & $\begin{array}{r}1.9 \\
2.6 \\
2.5 \\
5.6 \\
5.2 \\
11.1 \\
11.0\end{array}$ & $\begin{array}{r}2.2 \\
2.8 \\
2.5 \\
5.5 \\
5.0 \\
10.3 \\
10.3\end{array}$ & & $\begin{array}{r}0.6 \\
-0.2 \\
0.6 \\
-1.1 \\
-0.7 \\
-4.1 \\
-3.9\end{array}$ & $\begin{array}{r}0.2 \\
0.0 \\
0.4 \\
-0.6 \\
-0.3 \\
-1.8 \\
-1.8\end{array}$ & \\
\hline
\end{tabular}

(b)

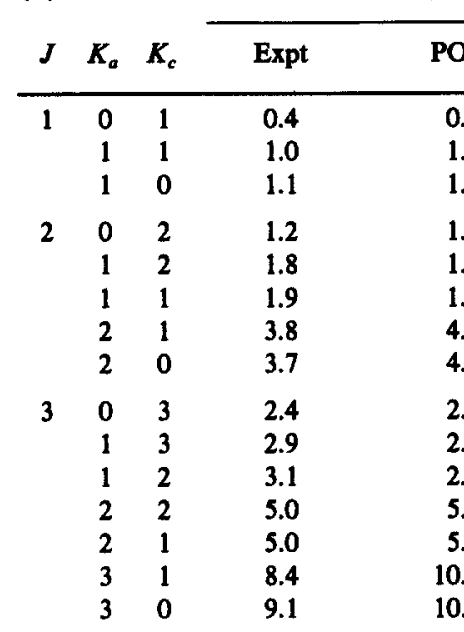

(110)

\begin{tabular}{ll} 
POS & Ref. \\
\hline 0.3 & 0.5 \\
1.3 & 0.8 \\
1.3 & 0.7 \\
1.0 & 1.5 \\
1.9 & 1.9 \\
1.9 & 1.6 \\
4.9 & 1.6 \\
4.8 & 1.8 \\
\hline
\end{tabular}

(011)

\begin{tabular}{rrrrrrr}
\hline & & & & & & \\
\cline { 6 - 7 } \cline { 5 - 6 } Expt & POS & Ref. 5 & & Expt & \multicolumn{1}{c}{ POS } & Ref. 5 \\
\hline 0.2 & 0.2 & -0.2 & & 0.2 & 0.1 & 0.7 \\
1.7 & 1.6 & 0.4 & & -0.7 & -0.3 & 0.4 \\
1.7 & 1.4 & 0.9 & & -0.6 & -0.1 & -0.2 \\
0.7 & 0.8 & -0.5 & & 0.5 & 0.2 & 2.0 \\
2.2 & 2.1 & 0.2 & & -0.4 & -0.2 & 1.7 \\
2.0 & 1.9 & 1.7 & & -0.1 & 0.0 & -0.1 \\
6.5 & 5.8 & 3.5 & & -2.7 & -0.9 & -1.9 \\
6.3 & 5.6 & 2.6 & & -2.6 & -0.8 & -0.8 \\
1.8 & 1.9 & & & 0.6 & 0.2 & \\
2.9 & 3.0 & & & 0.0 & -0.1 & \\
2.6 & 2.5 & & 0.5 & 0.3 & \\
6.0 & 6.4 & & -1.0 & -0.7 & \\
6.2 & 5.9 & & -1.2 & -0.5 & \\
14.0 & 12.5 & & -5.6 & -2.0 & \\
14.0 & 12.5 & & -4.9 & -2.0 & \\
& & & & & &
\end{tabular}

(c)

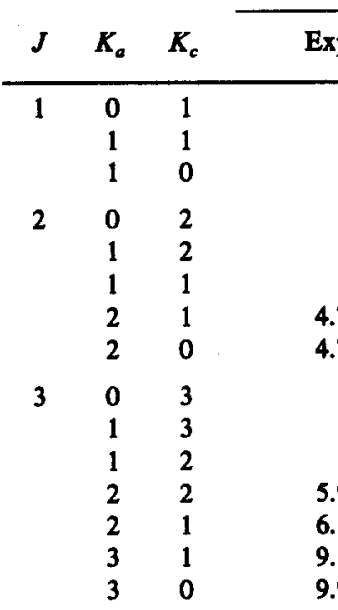

(120)

(021)

\begin{tabular}{cr} 
POS & Ref. 5 \\
\hline 0.2 & -1.6 \\
2.0 & 0.3 \\
1.9 & 0.9 \\
0.8 & -3.0 \\
2.6 & 0.8 \\
2.2 & 1.5 \\
7.4 & 2.7 \\
7.3 & 2.9 \\
1.8 & \\
3.4 & \\
2.8 & \\
8.1 & \\
7.5 & \\
16.0 & \\
16.0 &
\end{tabular}

1.6
0.3
0.9
-3.0
0.8
1.5
2.7
2.9

\begin{tabular}{cc}
\multicolumn{2}{c}{$v^{+}-v^{-}$} \\
\hline Expt & POS \\
& \\
& \\
& \\
& \\
-4.1 & -0.8 \\
-4.0 & -0.9 \\
& \\
& \\
-3.7 & -0.7 \\
-3.0 & -0.5 \\
-9.2 & -2.0 \\
-9.1 & -2.0
\end{tabular}


TABLE VIII (continued).

\begin{tabular}{|c|c|c|c|c|c|c|c|c|}
\hline \multirow{2}{*}{$\begin{array}{l}\text { (d) } \\
J\end{array}$} & \multirow[b]{2}{*}{$\boldsymbol{K}_{\boldsymbol{a}}$} & \multirow[b]{2}{*}{$\boldsymbol{K}_{\mathrm{c}}$} & \multicolumn{2}{|c|}{$(130)$} & \multicolumn{2}{|c|}{$(031)$} & \multicolumn{2}{|c|}{$v^{+}-v^{-}$} \\
\hline & & & Expt & POS & Expt & POS & Expt & POS \\
\hline 1 & $\begin{array}{l}0 \\
1 \\
1\end{array}$ & $\begin{array}{l}1 \\
1 \\
0\end{array}$ & $\begin{array}{l}0.4 \\
1.5 \\
1.5\end{array}$ & $\begin{array}{l}0.2 \\
2.6 \\
2.4\end{array}$ & $\begin{array}{l}0.2 \\
3.4 \\
3.3\end{array}$ & $\begin{array}{l}0.2 \\
2.8 \\
2.6\end{array}$ & $\begin{array}{r}0.2 \\
-1.9 \\
-1.8\end{array}$ & $\begin{array}{r}0.0 \\
-0.2 \\
-0.2\end{array}$ \\
\hline 2 & $\begin{array}{l}0 \\
1 \\
1 \\
2 \\
2\end{array}$ & $\begin{array}{l}2 \\
2 \\
1 \\
1 \\
0\end{array}$ & $\begin{array}{l}1.2 \\
2.2 \\
2.2 \\
6.0 \\
6.1\end{array}$ & $\begin{array}{l}0.8 \\
3.1 \\
2.8 \\
9.4 \\
9.3\end{array}$ & $\begin{array}{r}0.6 \\
3.7 \\
3.5 \\
12.8 \\
12.7\end{array}$ & $\begin{array}{r}0.7 \\
3.2 \\
2.8 \\
10.1 \\
10.0\end{array}$ & $\begin{array}{r}0.6 \\
-1.5 \\
-1.3 \\
-6.8 \\
-6.6\end{array}$ & $\begin{array}{r}0.1 \\
-0.1 \\
0.0 \\
-0.7 \\
-0.7\end{array}$ \\
\hline 3 & $\begin{array}{l}0 \\
1 \\
1 \\
2 \\
2 \\
3 \\
3\end{array}$ & $\begin{array}{l}3 \\
3 \\
2 \\
2 \\
1 \\
1 \\
0\end{array}$ & $\begin{array}{r}2.6 \\
3.5 \\
3.5 \\
7.2 \\
6.7 \\
13.2 \\
13.2\end{array}$ & $\begin{array}{r}1.9 \\
4.0 \\
3.5 \\
10.1 \\
9.7 \\
19.7 \\
19.7\end{array}$ & $\begin{array}{r}1.7 \\
4.8 \\
4.0 \\
13.4 \\
12.8 \\
27.2 \\
27.1\end{array}$ & $\begin{array}{r}1.7 \\
4.1 \\
3.4 \\
10.7 \\
10.2 \\
21.3 \\
21.3\end{array}$ & $\begin{array}{r}0.9 \\
-1.3 \\
-0.5 \\
-6.2 \\
-6.1 \\
-14.0 \\
-13.9\end{array}$ & $\begin{array}{r}0.2 \\
-0.1 \\
0.1 \\
-0.6 \\
-0.5 \\
-1.6 \\
-1.6\end{array}$ \\
\hline
\end{tabular}

TABLE IX. Differences between the rotational energies of states with two stretching quanta and corresponding states with no stretching excitation, for (left columns) no bending excitation and (right columns) one quantum of bending excitation.

\begin{tabular}{|c|c|c|c|c|c|c|c|c|c|c|c|c|}
\hline \multirow[b]{3}{*}{$J$} & \multirow[b]{3}{*}{$\boldsymbol{K}_{a}$} & \multirow[b]{3}{*}{$\boldsymbol{K}_{c}$} & \multicolumn{5}{|c|}{$v_{2}=0$} & \multicolumn{5}{|c|}{$v_{2}=1$} \\
\hline & & & \multicolumn{3}{|c|}{ Expt } & \multirow[b]{2}{*}{ POS } & \multirow[b]{2}{*}{ Ref. 5} & \multicolumn{3}{|c|}{ Expt } & \multirow[b]{2}{*}{ POS } & \multirow[b]{2}{*}{ Ref. 5} \\
\hline & & & $(200)$ & (101) & $(002)$ & & & $(210)$ & (111) & $(012)$ & & \\
\hline \multirow[t]{3}{*}{1} & 0 & 1 & 0.8 & 0.6 & 0.5 & 0.6 & 0.5 & 0.7 & 0.6 & 0.4 & 0.5 & 0.8 \\
\hline & 1 & 1 & 1.9 & 2.2 & 2.6 & 2.3 & 1.2 & 2.1 & 2.7 & 3.2 & 2.8 & 1.3 \\
\hline & 1 & 0 & 1.9 & 2.2 & 2.5 & 2.1 & 1.4 & 2.2 & 2.7 & 3.1 & 2.6 & 1.6 \\
\hline \multirow[t]{5}{*}{2} & 0 & 2 & 2.3 & 2.0 & 1.7 & 1.9 & 1.9 & 2.3 & 1.9 & 1.6 & 1.8 & 2.0 \\
\hline & 1 & 2 & 3.4 & 4.1 & 3.6 & 3.5 & 2.5 & 3.6 & 4.1 & 4.2 & 3.9 & 2.5 \\
\hline & 1 & 1 & 3.5 & 3.2 & 3.6 & 3.2 & 3.0 & 3.7 & 2.4 & 3.9 & 3.6 & 2.9 \\
\hline & 2 & 1 & 7.0 & 8.1 & 9.5 & 8.2 & 4.2 & 9.3 & 9.9 & 11.9 & 10.3 & 4.6 \\
\hline & 2 & 0 & 6.1 & 8.0 & 9.3 & 8.0 & 4.3 & 7.6 & 9.8 & 11.6 & 10.1 & 4.7 \\
\hline \multirow[t]{7}{*}{3} & 0 & 3 & 4.8 & 4.3 & 3.8 & 4.2 & & 4.7 & 4.2 & 3.7 & 4.0 & \\
\hline & 1 & 3 & 5.6 & 5.9 & 5.3 & 5.4 & & 5.9 & 6.1 & 5.8 & 5.8 & \\
\hline & 1 & 2 & 5.9 & 5.1 & 5.0 & 4.9 & & 6.0 & 5.0 & 5.3 & 5.1 & \\
\hline & 2 & 2 & 9.4 & 10.5 & 11.0 & 9.9 & & 10.9 & 12.0 & 13.2 & 11.9 & \\
\hline & 2 & 1 & 8.5 & 10.1 & 10.1 & 9.1 & & 9.6 & 11.4 & 12.3 & 11.0 & \\
\hline & 3 & 1 & 13.8 & 17.5 & & 17.9 & & 16.5 & 21.3 & 25.6 & 22.3 & \\
\hline & 3 & 0 & 13.9 & 17.5 & 20.5 & 17.9 & & 16.5 & 21.3 & 25.6 & 22.4 & \\
\hline
\end{tabular}

TABLE X. Differences in rotational energies of states with five stretching quanta and those with no stretching quanta. The experimental differences are obtained by subtraction of the values given by Rothman (Ref. 33) for the bands (302) and (401) from the frequencies reported by Antipov et al. (Ref. 31) for rotation-vibration transitions.

\begin{tabular}{ccccccc}
\hline & & & \multicolumn{3}{c}{ Expt } & \\
\cline { 4 - 6 }$J$ & $K_{a}$ & $K_{c}$ & $(302)$ & $(401)$ & POS \\
\hline 1 & 0 & 1 & 1.7 & 1.6 & 1.5 \\
& 1 & 1 & 3.7 & 3.6 & 5.3 \\
& 1 & 0 & 3.8 & 3.8 & 5.1 \\
2 & 0 & 2 & 4.9 & & 4.9 \\
& 1 & 2 & 6.9 & 6.8 & 8.4 \\
& 1 & 1 & 7.4 & 7.3 & 7.8 \\
& 2 & 1 & 12.0 & 11.5 & 19.6 \\
& 2 & 0 & 11.9 & 11.6 & 18.5 \\
3 & 0 & 3 & 11.0 & 11.0 & 10.8 \\
& 1 & 3 & 11.9 & 11.5 & 13.3 \\
& 1 & 2 & 12.6 & 13.1 & 13.5 \\
& 2 & 2 & 16.7 & 16.1 & 23.8 \\
& 2 & 1 & 16.5 & 15.9 & 21.6 \\
& 3 & 1 & 18.6 & & 42.5 \\
& 3 & 0 & 18.6 & 18.9 & 42.3 \\
\hline \hline
\end{tabular}

Our work thus gives rise to the new approach to calculating rotation-vibrational spectra of water-like molecules with excitation in local modes. After it was completed, we learned of very accurate vibrational calculations carried out by Chen, Maessen, and Wolfsberg ${ }^{35}$ for the ground and one-quantum excited vibrational state of $\mathrm{H}_{2} \mathrm{O}$. These calculations essentially exploit the normal-mode basis set and hence can only be extended to states with several quanta in stretching modes by using a large number of basis wave functions. Either quantization of the problem in a space-fixed frame ${ }^{17}$ or using the Eckart Hamiltonian expressed in terms of geometrical coordinates ${ }^{36}$ seem to be more effective alternatives to the aforementioned expansion of the normal-mode basis set for describing high vibrational states.

\section{ACKNOWLEDGMENTS}

This research was supported in part by a Grant from the National Science Foundation. G.D.-B. also wishes to express thanks for a Grant from the U.S.-Spain Joint Committee for Scientific and Technological Cooperation. 
'G. A. Natanson, G. S. Ezra, G. Delgado-Barrio, and R. S. Berry, J. Chem. Phys. 81, 3400 (1984).

${ }^{2}$ J. T. Hougen, P. R. Bunker, and J. W. C. Johns, J. Mol. Spectrosc. 34, 136 (1970).

${ }^{3}$ P. R. Bunker and J. M. R. Stone, J. Mol. Spectrosc, 41, 310 (1972).

${ }^{4}$ P. R. Bunker and B. M. Landsberg, J. Mol. Spectrosc. 67, 376 (1977).

sJ. R. Reimers and R. O. Watts, Mol. Phys. 52, 357 (1984).

${ }^{6}$ R. T. Lawton and M. S. Child, Mol. Phys. 37, 1799 (1979).

${ }^{7}$ G. S. Exra and R. S. Berry, Phys. Rev. A 25, 1513 (1982).

${ }^{8}$ B. R. Johnson, R. T. Skodje, and W. P. Reinhardt, Chem. Phys. Lett. (in press).

${ }^{9}$ G. S. Ezra, Chem. Phys. Lett. 101, 259 (1983).

${ }^{10}$ G. A. Natanson, G. Delgado-Barrio, and R. S. Berry (to be published).

${ }^{11} R$. Radau, Ann. Sci. Ecole Normale Superior 5, 311 (1868).

${ }^{12}$ M. N. Adamov and G. A. Natanson, Vestn. Leningr. Univ., No. 22, 30 (1970), in Russian.

${ }^{13}$ F. T. Smith, Phys. Rev. Lett. 45, 1157 (1980).

${ }^{14}$ B. R. Johnson, Wisc. Theor. Chem. Inst. Rept. WIS-TCI-650 (1981).

${ }^{15}$ E. Pollak and R. E. Wyatt, J. Chem. Phys. 81, 1801 (1984).

${ }^{16}$ G. A. Natanson and R. S. Berry (to be published).

${ }^{17}$ G. A. Natanson and R. S. Berry (in preparation).

${ }^{18}$ G. S. Ezra and R. S. Berry, Phys. Rev. A 28, 1989 (1983).

${ }^{19}$ M. S. Child and R. T. Lawton, Chem. Phys. Lett. 87,217 (1982).

${ }^{20}$ E. B. Wilson, Jr. and J. B. Howard, J. Chem. Phys. 4, 260 (1936).
${ }^{21}$ J. K. G. Watson, Mol. Phys. 15, 479 (1968).

${ }^{22}$ (a) G. A. Natanson, Mol. Phys. 46, 481 (1982); (b) Studies in Physics d Theoretical Chemistry (Elsevier, Amsterdam, 1983), Vol. 23, p. 201.

${ }^{23}$ G. P. Carney, L. A. Curtiss, and S. R. Langhoff, J. Mol. Spectrosc. 61, 371 (1976).

24P. Botschwina, Chem. Phys. 40, 33 (1979).

${ }^{25}$ J.-M. Flaud and C. Camy-Peyret, J. Mol. Spectrosc. 51, 142 (1974).

${ }^{26}$ J.-M. Flaud, C. Camy-Peyret, and J.-P. Maillard, Mol. Phys. 32, 499 (1976).

${ }^{27}$ C. Camy-Peyret and J.-M. Flaud, Mol. Phys. 32, 523 (1977).

${ }^{28}$ R. A. Toth and J. S. Margolis, J. Mol. Spectrosc. 55, 229 (1975).

${ }^{29} \mathrm{C}$. Camy-Peyret and J.M. Flaud, J. Mol. Spectrosc. 59,327 (1976).

${ }^{30}$ J.-M. Flaud, C. Camy-Peyret, K. N. Rao, D.-W. Chen, Y.-S. Hoh, and J.-P. Maillard, J. Mol. Spectrosc. 75, 339 (1979).

${ }^{31}$ A. B. Antipov, A. D. Bykov, V. E. Zuev, V. A. Kapitano, V. P. Lopasov, Yu. S. Makushkin, V. I. Tolmachev, and O. N. Ulenikov, Opt. Spectrosc. Wash. 53,613 (1982).

${ }^{32}$ (a) R. Mecke, Z. Phys. 81, 313 (1933); (b) W. Baumann and R. Mecke, ibid. 81, 445 (1933); (c) K. Freudenberg and R. Mecke, ibid. 81, 465 (1933).

${ }^{33}$ L. S. Rothman, Appl. Opt. 17, 3517 (1978).

${ }^{34}$ According to the first two columns of Table IV these are states of $05^{+}$and $05^{-}$in the local mode description of energy levels.

${ }^{35}$ C.-L. Chen, B. Mäessen, and M. Wolfsberg, J. Chem. Phys. 83, 1795 (1985).

${ }^{36}$ G. A. Natanson, Mol. Phys. (submitted). 